\title{
Cardioprotection of ischemic preconditioning in rats involves upregulating adiponectin
}

\author{
Hui Wang', Wenjing Wu2, Jun Duan', Ming Ma3, Wei Kong4, Yuannan Ke², Gang Li1 \\ and Jingang Zheng ${ }^{2}$
}

'Department of Intensive Care Unit, China-Japan Friendship Hospital, Beijing, People's Republic of China 2Department of Cardiology, China-Japan Friendship Hospital, Beijing, People's Republic of China

${ }^{3}$ Department of Plastic and Cosmetology, Beijing Haidian Hospital, Beijing, People's Republic of China ${ }^{4}$ Department of Physiology and Pathophysiology, School of Basic Medical Sciences, Peking University, Key Laboratory of Molecular Cardiovascular Science, Ministry of Education, Beijing, People's Republic of China

Correspondence should be addressed to J Zheng

Email

victorzheng@sina.com

\begin{abstract}
It has been reported that ischemic preconditioning (IPC) and adiponectin (APN) are cardioprotective in many cardiovascular disorders. However, whether APN mediates the effect of IPC on myocardial injury has not been elucidated. This study was conducted to investigate whether IPC affects myocardial ischemic injury by increasing APN expression. Male adult rats with cardiac knockdowns of APN and its receptors via intramyocardial small-interfering RNA injection were subjected to IPC and then myocardial infarction (MI) at $24 \mathrm{~h}$ after IPC. Globular APN (gAd) was injected at $10 \mathrm{~min}$ before MI. APN mRNA and protein levels in myocardium as well as the plasma APN concentration were markedly high at 6 and $12 \mathrm{~h}$ after IPC. IPC ameliorated myocardial injury as evidenced by improved cardiac functions and a reduced infarct size. Compared with the control MI group, rats in the IPC + MI group had elevated levels of left ventricular ejection fraction and fractional shortening and a smaller $\mathrm{MI}$ size $(P<0.05)$. However, the aforementioned protective effects were ameliorated in the absence of APN and APN receptors, followed by the inhibition of AMP-activated protein kinase (AMPK) phosphorylation, but reversed by gAd treatment in wild-type rats, and AMPK phosphorylation increased $(P<0.05)$. Overall, our results suggest that the cardioprotective effects of IPC are partially due to upregulation of APN and provide a further insight into IPC-mediated signaling effects.
\end{abstract}
Key Words
- myocardial infarction
- ischemia preconditioning
- adiponectin
- gene regulation

Journal of Molecular

Endocrinology

(2017) 58, 155-165

\section{Introduction}

Ischemic preconditioning (IPC) is a well-known phenomenon in which brief intermittent periods of ischemia-reperfusion protect the myocardium against subsequent long and lethal ischemia. It is also known that the prognosis after myocardial infarction (MI) is better for patients with unstable angina than those suffering an unpredicted MI. These fundamental observations suggest that endogenous mechanisms of IPC exist in the heart to protect against future ischemic events. Many studies have attempted to define the signaling mechanisms induced by IPC with the goal of developing pharmacological mimics to provide protection. Such studies have found that one or more factors released in response to brief episodes of ischemia during IPC interact with receptors, locally or at a http://jme.endocrinology-journals.org DOI: 10.1530/JME-16-0163
C 2017 Society for Endocrinology Printed in Great Britain 
distance, triggering the protective response (e.g. a reduced infarct size, recovery of contractile functions, antiarrhythmia and angiogenesis) (Crisostomo et al. 2006, Evrengul et al. 2006, Andersen et al. 2012). Nevertheless, the underlying mechanisms are still not fully understood. A modern experimental strategy for treating myocardial ischemia is to induce neovascularization of the heart by angiogens. Previous studies by us as well as others have reported that IPC mediates angiogenic effects via growth factors and receptor stimulation in endothelial cells during MI (Fukuda 2004, Zheng et al. 2006, Thirunavukkarasu et al. 2008). Therefore, the identification of key factors or angiogens has a potential benefit for the development of pharmacological therapies for heart injuries induced by acute MI.

Adiponectin (APN) is an adipocyte-derived cytokine normally present in human plasma at concentrations of $0.5-30 \mu \mathrm{g} / \mathrm{mL}$, comprising about $0.01 \%$ of all plasma proteins (Hotta et al. 2000). In addition to its well-defined insulin sensitization and metabolic regulatory effects, APN increases the phosphorylation of AMP-activated protein kinase (AMPK) that has vascular protective, antiinflammatory and anti-ischemic effects (Ling et al. 2007). Numerous epidemiological studies have shown that reduced APN levels correlate with an increased risk of cardiovascular disease (Goldstein et al. 2009), whereas high plasma APN concentrations are associated with a lower risk of MI in men (Pischon et al. 2004). In vivo experiments have also demonstrated that increasing plasma APN protects the myocardium against ischemic injury (Shibata et al. 2005, Ling et al. 2007). A proteolytic cleavage product of APN, which includes the globular domain, also exists in plasma biologically. Globular APN (gAd) administration protects the myocardium against ischemia-reperfusion injury, involving a reduction of oxidative/nitrative stress and inhibition of endoplasmic reticulum stress (Jia et al. 2013, Song et al. 2014). Therefore, enhancing APN production has been accepted as a potential therapeutic modality for amelioration of cardiovascular injury. Although APN has been regarded to be of exclusive adipocyte origin, interestingly, cardiomyocytes also synthesize and secrete biologically active APN (Pineiro et al. 2005), and such cardiac-derived APN protects against myocardial ischemia/reperfusion injury and has a peroxisome proliferator-activated receptor- $\gamma$-mediated antihypertrophic effect (Amin et al. 2010, Wang et al. 2010b). These findings indicate that endogenous cardiac-derived APN may exert its cardioprotective effect through paracrine/ autocrine activation.

Some studies have also implicated APN in angiogenesis (Ouchi et al. 2004, Shibata et al. 2004). A recent study reported that gAd induces endothelial cell proliferation as well as in vitro migration and stimulates the release of endothelial angiogenic factors (Raghu et al. 2012). As a kind of angiogenic factor, whether APN is a mediator of IPC in cardioprotective effects remains unknown. Data from our previous study have demonstrated that IPC upregulates myocardial expression of APN at mRNA and protein levels and increases the plasma APN concentration (Wang et al. 2010a). Here, we hypothesized that the increase in myocardium and plasma APN levels induced by IPC results in protection against ischemia. Therefore, the aims of the current study are (1) to delineate the potential causative relationship between IPC-induced APN upregulation and the resultant cardioprotective effects and (2) to investigate the underlying mechanisms using myocardial delivery of small-interfering RNA (siRNA) and administration of human recombinant gAd.

\section{Materials and methods}

\section{Experimental protocols}

All experiments were performed with adherence to the National Institute of Health's Guide for the Use and Care of Laboratory Animals and were approved by the Committee on Animal Care and Use of the China-Japan Friendship Hospital (Beijing, China). Male SpragueDawley rats (8 weeks of age, weighing $300 \pm 50$ g) were obtained from Vital River Laboratory Animal Technology Co. Ltd. (Beijing, China). Rats were randomized to receive IPC or a sham procedure. At $24 \mathrm{~h}$ after IPC, some rats in both groups were subjected to MI for cardiac function and infarct size determinations. To investigate the potential causative relationship between APN- and IPCinduced cardioprotective effects and the further signaling activation of APN, rats were injected with APN or APN receptor (AdipoR) 1 and 2 siRNAs (Sugano et al. 2005) in their myocardium at $10 \mathrm{~min}$ before IPC or administered with $4 \mu \mathrm{g} / \mathrm{g}$ gAd (Sigma) at $10 \mathrm{~min}$ before MI (Ling et al. 2007). All surgical procedures as well as blood and cardiac tissue collections were performed under anesthesia to avoid stress.

\section{IPC and MI model in rats}

Animals were divided into IPC and sham groups, which were randomized to receive IPC or a sham procedure according to our previously published protocol (Zheng et al. 2006), with slight modifications. In brief, after anesthesia with $1 \%$ pentobarbital sodium, a tracheotomy

Published by Bioscientifica Ltd. 
was performed, and then the animal was intubated and ventilated with air. A left intercostal thoracotomy was performed at the fourth and fifth intercostal space. Upon identification of the left anterior descending artery (LAD), a 7-0 suture was secured at $2 \mathrm{~mm}$ below the level of the tip of the normally positioned left auricle, and four rounds of 6-min ischemia and reperfusion were induced to achieve minimal mortality, which differed from our previous ischemia protocol with high mortality (Zheng et al. 2006). The success of ischemic induction was verified by a color change in the cardiac surface from reddish to pale and continuous electrocardiographic monitoring. The recovered reddish color and the appearance of ventricular arrhythmia indicated successful reperfusion. After the conclusion of the fourth ischemia-reperfusion cycle, the suture was removed, the chest wall was closed and the animal was removed from the ventilator and placed under a heat lamp to recover. Sham-operated control rats underwent the same surgical procedures except the LAD was subjected to transient occlusion.

Additional sets of rats, which underwent repeated operations after sham or IPC procedures, had a permanently ligated LAD. Briefly, at $24 \mathrm{~h}$ after IPC or sham operation, the rats were re-anesthetized and their LAD was permanently ligated with 8-0 nylon sutures, which are indicated as IPC+MI or MI, respectively. The chest wall was closed, and the rats were returned to their cages and fed routinely for 2 weeks.

All rats were killed under anesthesia. Rats in IPC and sham groups were killed at $0,6,12$ or $24 \mathrm{~h}$ after the procedure ( $n=14$ for each time point). Blood samples $(3 \mathrm{~mL})$ were collected in BD EDTA anticoagulant tubes from the abdominal aorta. The tubes were centrifuged at $3000 \mathrm{~g}$ for $10 \mathrm{~min}$ at room temperature, and plasma samples were frozen as quickly as possible at $-80^{\circ} \mathrm{C}$ for storage. Their hearts were excised, snap-frozen in liquid nitrogen or embedded in OCT, fixed for immunohistochemistry and stored at $-80^{\circ} \mathrm{C}$ until processing. At 14 days after ligation, before the hearts of rats in MI and IPC+MI groups were harvested for histological analysis, M-mode echocardiograms were recorded ( $n=12$ in each group).

\section{siRNA transfection in vivo}

siRNA-mediated gene silencing was used to knockdown APN, AdipoR1 and AdipoR2 in the rat heart. Specific siRNAs were designed using Block-iT RNAi Designer and chemically modified by the manufacturer (Invitrogen). A scramble Stealth RNAi duplex (Cat. No. 45-2001,
Invitrogen) served as a negative control. Briefly, rats were anesthetized with $1 \%$ pentobarbital sodium, and their heart was exposed via a left thoracotomy at the fifth intercostal space. According to a previously described method (Sugano et al. 2005), $200 \mu \mathrm{L}$ of siRNA solution $(20 \mu \mathrm{mol} / \mathrm{L})$ at a final dosage of $0.5 \mu \mathrm{g} / \mathrm{g}$ and in vivo jetPEI (Polyplus-transfection, Bioparc, France) (ratio: $8 \mu \mathrm{L} / 50 \mu \mathrm{g}$ siRNA) were delivered via intramyocardial injections at three symmetrical points following two sides of the anterior descending branch to avoid injecting heart chambers or the artery lumen as described previously (Zheng et al. 2006). Successful injection was confirmed by a local swollen and pale myocardium. Rats were processed for further sham operation, IPC or IPC + MI protocols after the exposure period ( $n=8$ in each group).

\section{gAd injection}

Ten minutes before MI, human recombinant gAd ( $4 \mu \mathrm{g} / \mathrm{g}$; Sigma) was administered intraperitoneally to anesthetized IPC rats, and saline was administrated to rats as the control group ( $n=8$ in each group). It has been previously reported that no human APN is detected before gAd administration, intraperitoneally administered gAd $(2 \mu \mathrm{g} / \mathrm{g})$ is partially absorbed and human APN is detected at 10, 60 and $120 \mathrm{~min}$ after injection (Ling et al. 2007).

\section{Determination of cardiac functions and the MI size}

After inducing anesthesia by intraperitoneal injection of $1 \%$ pentobarbital sodium, the heart rate of the rat was kept at 350-400 beats/min, and cardiac functions were determined via echocardiography (Vivid E9, GE Healthcare) by evaluating the left ventricular ejection fraction (LVEF\%) and fractional shortening (FS\%) at 14 days after MI. FS\% and LVEF\% were acquired by measuring the average left ventricular end-diastolic diameter (LVDd) and left ventricular end-systolic diameter (LVDs) for three cardiac cycles by the formula $\mathrm{FS}(\%)=((\mathrm{LVDd}-\mathrm{LVDs}) / \mathrm{LVDd}) \times 100 \%$, and $\quad$ LVEF $(\%)=\left(\left(\operatorname{LVDd}^{3}-\mathrm{LVDs}^{3}\right) / \mathrm{LVDd}^{3}\right) \times 100 \%$.

After completing the functional determinations, the hearts were harvested and stained. Briefly, the left ventricle was sectioned into six equal transverse slices starting from the apex to base. Sections at the level of the midpapillary muscle were prepared at $6 \mu \mathrm{m}$ thicknesses and stained with hematoxylin and eosin and aldehyde fuchsin Gomori's trichrome. The myocardial infarction size was scored using the Universal Image-1 video

Published by Bioscientifica Ltd. 
image analyzer system and expressed as a percentage of the total left ventricular myocardial area as described previously (Zheng et al. 2006). After staining, the infarcted myocardium is blue because of replacement by collagen fibers, whereas normal myocardium is reddish.

\section{Quantitative real-time PCR analysis}

Total RNA was extracted from rat hearts using TRIzol (Life Technologies) according to the manufacturer's instructions. The RNA concentration was determined by the absorbance at 260 and $280 \mathrm{~nm}$. Total RNA was reverse transcribed into cDNA using a PrimeScript RT-PCR Kit (Takara Bio). Primers were designed as follows: 5'-AATCCTGCCCAGTCATGAAG-3' and 5'-TCTCCAGGAGTGCCATCTCT-3' for rat APN; 5'-CCCTCCACCCAAGGAAACT-3' and 5'-GCCCTACGCTGAATGCTGA-3' for rat glyceraldehyde3-phosphate dehydrogenase (GAPDH). Real-time PCR amplification was performed using SYBR Premix Ex Taq (Perfect Real Time) (Takara Bio). The reactions were carried out at $94^{\circ} \mathrm{C}$ for $3 \mathrm{~min}$, followed by 35 cycles at $94^{\circ} \mathrm{C}$ for $30 \mathrm{~s}, 54-62^{\circ} \mathrm{C}$ for $30 \mathrm{~s}$ and $72^{\circ} \mathrm{C}$ for $30 \mathrm{~s}$, and finally $72^{\circ} \mathrm{C}$ for $10 \mathrm{~min}$ (PRISM 7300, Applied Biosystems). Expression of APN was normalized to GAPDH expression. Relative expression was determined using the $2^{-\Delta \Delta \mathrm{Ct}}$ method.

\section{Western blot analysis}

Frozen tissues $(100 \mathrm{mg})$ from each sample were homogenized for $10 \mathrm{~min}$ with tissue protein lysis buffer (Beyotime Institute of Biotechnology, Haimen, China) using a homogenizer, chilled on ice for $30 \mathrm{~min}$, and then centrifuged at $10,000 \mathrm{~g}$ for $5 \mathrm{~min}$ at $4^{\circ} \mathrm{C}$. The protein concentration was determined using a protein assay kit (BCA, Pierce Chemical). The same amount of protein $(50 \mu \mathrm{g})$ was separated by $8 \%$ sodium dodecyl sulfatepolyacrylamide gel electrophoresis and then transferred to a polyvinylidene fluoride membrane (Jianglai Science and Technology Co., Ltd., Shanghai, China). The membrane was blocked with $5 \%$ bovine serum albumin for $1 \mathrm{~h}$ at room temperature, followed by incubation with primary antibodies against APN, AdipoR1, AdipoR2, phosphorylated AMPK or total AMPK (Abcam plc) at $4^{\circ} \mathrm{C}$ overnight. Then, the membrane was incubated with a corresponding horseradish peroxidase-conjugated secondary antibody (ZSGB-Bio, Beijing, China) for $2 \mathrm{~h}$ at room temperature. Proteins were detected using an enhanced chemiluminescence reagent (SuperSignal West Pico, Pierce Chemical). Band intensity was quantified using the Odyssey infrared imaging system (LI-COR Biosciences, Lincoln, NE, USA). Protein expression was normalized to that of translation initiation factor 5 (Santa Cruz Biotechnology). Quantitative analysis was performed using ImageJ software (National Institutes of Health).

\section{Immunohistochemistry}

Consecutive frozen sections of heart were immunostained with an anti-APN antibody (Abcam plc) to examine APN expression at $24 \mathrm{~h}$ after IPC. Six rat heart sections 3-4 $\mu \mathrm{m}$ in thickness were analyzed in each group. Sections were examined by blinded analysis (Edelberg et al. 2002). The mean density of APN expression in the ischemic myocardium was analyzed by IPP 6.0 software (Zheng et al. 2006).

\section{Quantification of the plasma APN concentration}

Plasma APN levels were measured using an enzyme-linked immunosorbent assay (ELISA) for total rat APN (Rat Total Adiponectin ELISA kit; R\&D Systems). Optical density was measured at $450 \mathrm{~nm}$. The lowest detectable limit of the APN concentration was $0.004 \mathrm{ng} / \mathrm{mL}$ according to the manufacturer, and the coefficient of variation was $<10 \%$. To measure circulating APN in plasma, we diluted each sample 1000-fold in the diluent provided by the manufacturer. The APN concentration was then determined according to the manufacturer's instructions.

\section{Statistical analysis}

All continuous data are presented as the mean \pm standard error of the mean (s.E.M.). The protein band density was normalized to the corresponding loading control and then to the mean of the corresponding control group. One-way analysis of variance was used to compare more than two groups. The Student's $t$-test was used to analyze differences between two groups. Statistical analysis was performed using GraphPad Prism 5.0 (GraphPad Software). $P<0.05$ was considered as statistically significant.

\section{Results}

IPC ameliorates myocardial ischemic injury and increases cardiac-derived APN and plasma APN levels

Similar to our previous study (Wang et al. 2010a), we again verified the cardioprotective effects of IPC and the increased level of APN in myocardium and plasma regulated by IPC. To determine the cardioprotective effects,

Published by Bioscientifica Ltd 
we evaluated cardiac functions by echocardiography (LVEF\% and FS\%) and determined the myocardial infarct size at 14 days after permanent ligation of the LAD. IPC significantly enhanced the survival of MI rats by $60 \%$. IPCtreated hearts had better cardiac functions than the control MI group (increases in LVEF\% and FS\%) and a reduced infarct size (data shown in Supplementary material, see section on supplementary data given at the end of this article). Next, we detected the mRNA and protein levels of cardiac APN and the plasma APN concentration. Similar to our previous results, the expression of APN induced by IPC in the ischemic tissue was greatly upregulated at $6 \mathrm{~h}$, peaked at $12 \mathrm{~h}$ and decreased at $24 \mathrm{~h}$. Plasma APN levels increased at $6 \mathrm{~h}$ after IPC and reached their peak at $24 \mathrm{~h}$ after IPC (data shown in Supplementary material). Therefore, we confirmed the findings that cardiac-derived APN was induced and plasma APN levels had increased after IPC.

\section{Cardioprotective effects of IPC are partially mediated by increasing APN expression}

IPC exerted cardioprotective effects along with increased APN levels. Therefore, we delineated the potential causative relationship between IPC-induced APN upregulation and the resultant cardioprotective effects. To this end, we first knocked down APN levels in the heart in vivo by cardiac microinjection of siRNA. The APN protein level was downregulated to $60 \%$ at $24 \mathrm{~h}$ compared with scramble siRNA and the control IPC group (Fig. 1A and $\mathrm{B}$ ), indicating the feasibility of the method.

After decreasing the expression of APN by intramyocardial siRNA delivery, we analyzed the protective effect of IPC on MI. Compared with the control group, the delivery of APN siRNA greatly abolished the protective effect of IPC against MI. The myocardial infarct size was larger with APN siRNA after IPC than that with scramble siRNA (Fig. 1C and D, $30.17 \pm 1.29 \%$ vs $22.35 \pm 2.44 \%, P<0.05)$. In agreement with histological observations, the elevated LVEF\% and FS\% by preconditioning were ameliorated by cardiac APN silencing to a level comparable to wild-type hearts $(\mathrm{LVEF} \%, \quad 45.11 \pm 3.14 \%$ vs $56.89 \pm 2.74, P<0.05 ; \mathrm{FS} \%$, $18.64 \pm 1.60$ vs $24.40 \pm 1.76, P<0.05$ ) (Fig. $1 \mathrm{E}, \mathrm{F}$ and $G)$, with significant changes in other cardiac structural parameters (Table 1). Unsurprisingly, infusing exogenous human recombinant gAd significantly improved cardiac functions and reduced the infarct size compared with the vehicle (Fig. 2A, B, C, D and E). These data showed that the cardioprotective effects of IPC are weakened by cardiac APN silencing and strengthened by exogenous gAd administration, indicating that its effects are partially mediated by APN.
A
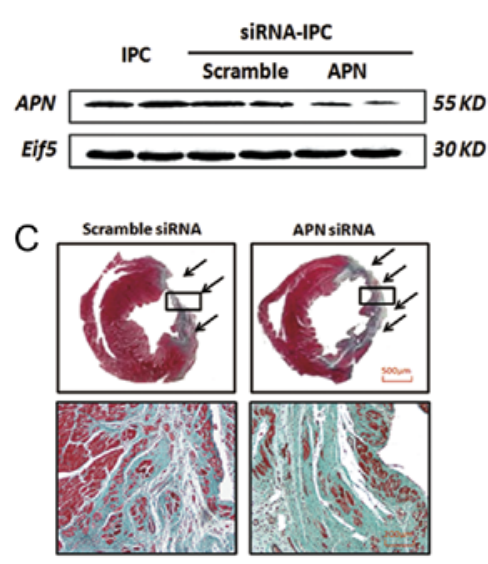

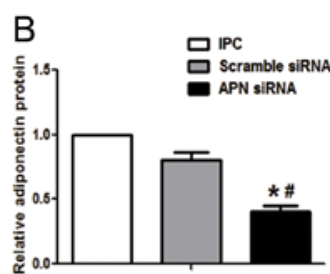

D

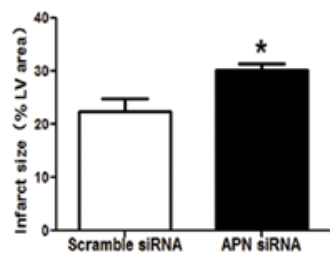

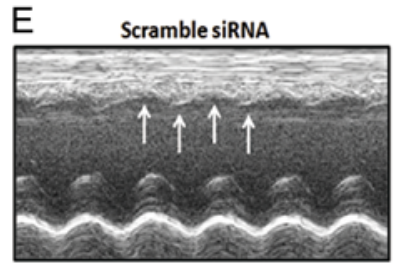

$\mathrm{F}$

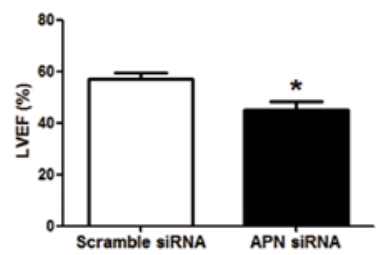

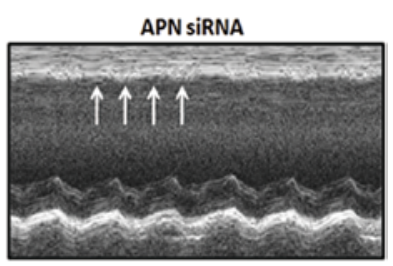

G

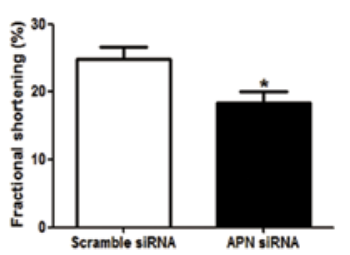

Figure 1

The cardioprotective effect of IPC was weakened by knockdown of APN through intramyocardium-delivered siRNA. (A) The rats were transfected with specific siRNA products for APN or negative control (scramble) siRNA by intramyocardial injection 10 min before IPC, expression of myocardial APN was determined by Western blot. (B) Quantitative analysis of Western blot showed APN expression. (C) Representative Masson's trichrome staining of rat hearts 14 days after MI (acute LAD ligation) $24 \mathrm{~h}$ after IPC with APN siRNA or scramble siRNA delivery. Arrows, transmural myocardial injury. (D) Measurement of cardiac injury (\% left ventricular area) in APN knockdown rats. (E) Representative echocardiography images from scramble or APN siRNA delivery rats 14 days after MI showed hypokinesis of left ventricular anterior wall in APN knock down rats (as arrow indicated). Left ventricular ejection fraction (LVEF\%) (F) and left ventricular fractional shortening (FS\%) (G) was determined from APN knock down rats by echocardiography 14 days after MI. $n=6-8$ animals/group. ${ }^{*} P<0.05$ vs scramble siRNA, ${ }^{\#} P<0.05$ vs WT rats.

http://jme.endocrinology-journals.org DOI: 10.1530/JME-16-0163
๑ 2017 Society for Endocrinology Printed in Great Britain
Published by Bioscientifica Ltd 

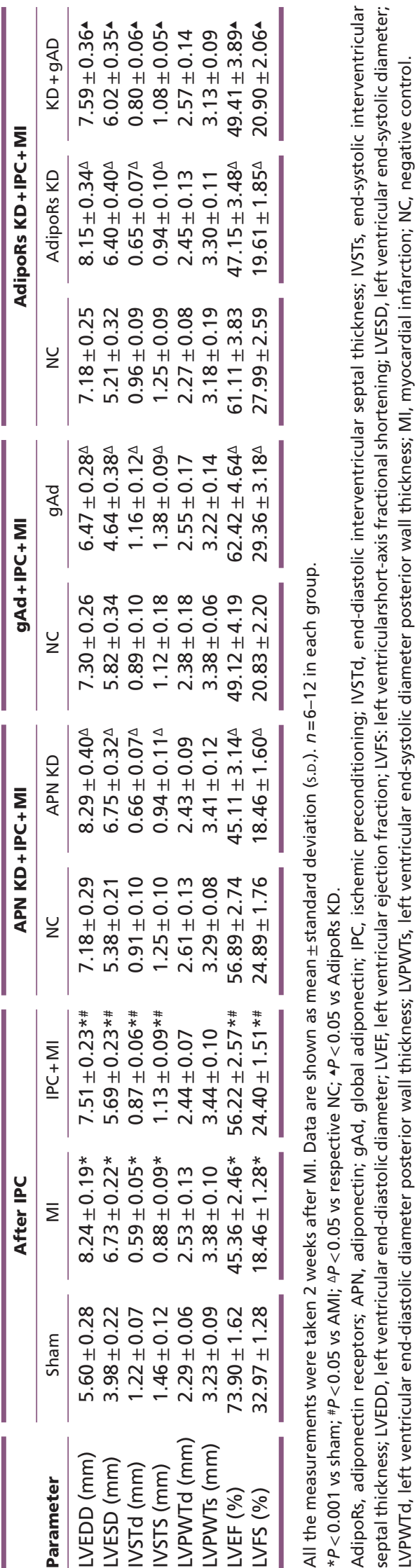

http://jme.endocrinology-journals.org DOI: 10.1530/JME-16-0163

() 2017 Society for Endocrinology Printed in Great Britain
AdipoR1 and AdipoR2 knockdowns in rats attenuate the cardioprotective effects of IPC

To further investigate the relationship between APN signaling activation and the cardioprotective effects of IPC, siRNA delivery was used to knockdown AdipoR1 and AdipoR2 in the myocardium in vivo. Intramyocardial siRNA delivery suppressed AdipoR1 and AdipoR2 expression to $64 \%$ in knockdown animals compared with scramble siRNA-injected animals (wild-type animals, WT) (Fig. 3A, B and C). Next, we determined the cardiac functions and infarct size. The results showed that the cardioprotective effects of IPC in AdipoR-knockdown rats were attenuated compared with wild-type animals (Figs 3D and $4 \mathrm{H}$, reduced LVEF\%, WT $61.11 \pm 3.83 \%$ vs $47.15 \pm 3.48 \%, \quad P<0.05 ; \quad$ FS $\%$ : WT $27.99 \pm 2.59 \%$ vs $19.61 \pm 1.85 \%, \quad P<0.05$; increased infarct size: WT $21.24 \pm 1.99 \%$ vs $29.89 \pm 2.02 \%, \quad P<0.05)$. The cardioprotective effects of administering gAd in AdipoR knockdown rats showed no significant improvement (Figs 3D and 4H). These data suggest that loss of APN receptor activation weakens the cardioprotective effects of IPC.

\section{IPC exerts myocardium-protective effects partly via AdipoR-AMPK signaling}

Our present study showed that the upregulated cardiac APN expression induced by IPC may contribute to the attenuation of myocardial ischemic injury. Because AMPK is the major downstream signaling molecule known to be partially responsible for cardioprotection by APN and cardioprotective effects of IPC are weakened by decreased AdipoR expression, we next determined the mechanism underlying the cardioprotective effects induced by IPC with particular focus on APN/AdipoR/ AMPK signaling. As illustrated in Fig. 4, we first determined phosphorylation of AMPK in wild-type hearts by Western blotting. The results showed that, compared with the MI group, IPC significantly increased the phosphorylation of AMPK $(2.20 \pm 0.22$ vs $1.49 \pm 0.18$, $P<0.05)$, which was significantly enhanced by gAd treatment $(3.01 \pm 0.28$ vs $2.20 \pm 0.22, P<0.05)$ (Fig. $4 \mathrm{~A}$ ). Next, we knocked down the expression of AdipoR and found that the aforementioned increase of AMPK phosphorylation was partly attenuated by treatment with IPC or gAd (Fig. 4B). These results suggest that IPC exerts myocardium-protective effects partly by the activation of APN/AdipoR/AMPK signaling.

Published by Bioscientifica Ltc. 

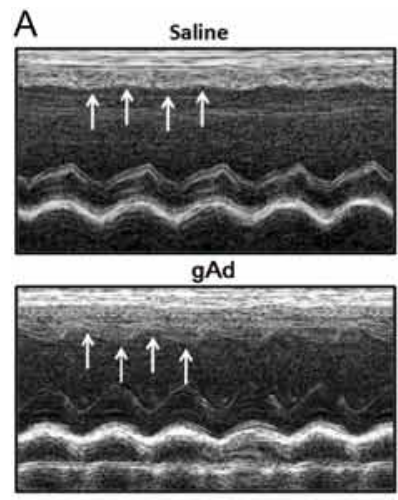

D

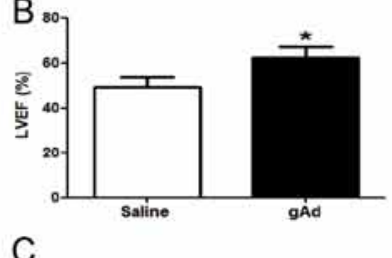

C

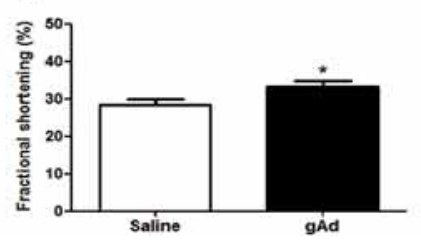

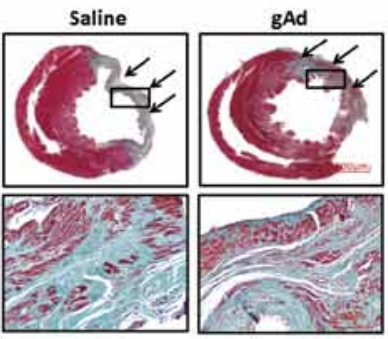

E

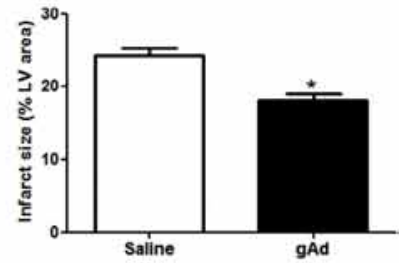

Figure 2

The cardioprotective effect of IPC was strengthened by exogenous gAd administration. (A) Representative echocardiography images from gAd administered rats 14 days after MI showed gAd administration improved systolic function of left anterior wall (as arrow indicated). (B) and (C) quantification of echocardiography from gAd administered rats by echocardiography 14 days after MI. (D) Exogenous gAd administration decreased infarct size as demonstrated by representative Masson's trichrome staining of rat hearts with gAd or saline administration. (E) Measurement of cardiac injury (\% left ventricular area) in gAd administrated rats. $n=6-8$ animals/group. ${ }^{*} P<0.05$ vs saline injected rats.

A

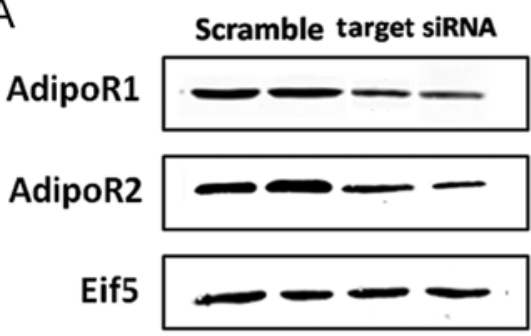

D

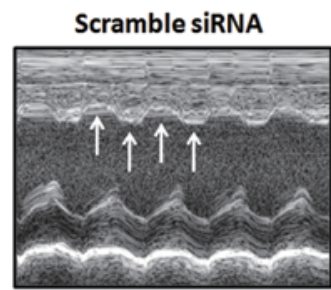

B

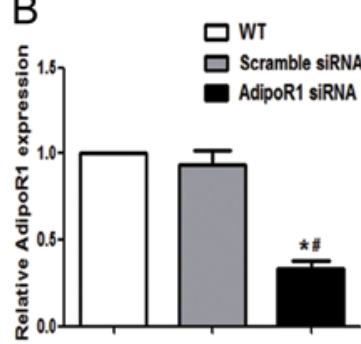

AdipoRs siRNA + gAd

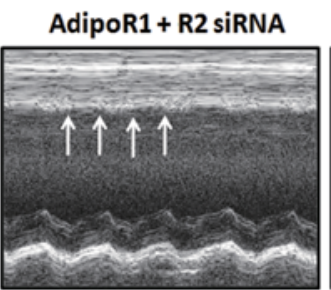

$\mathrm{F}$
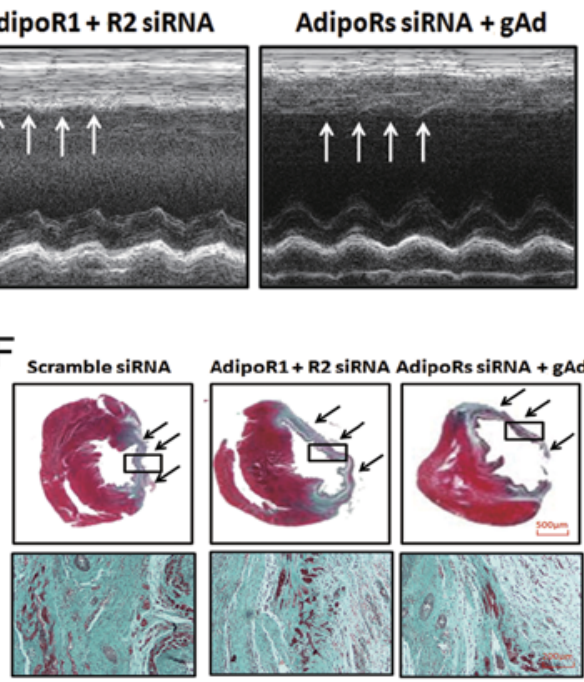

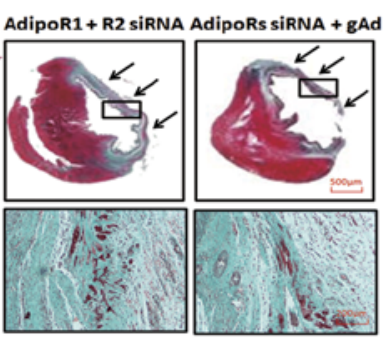

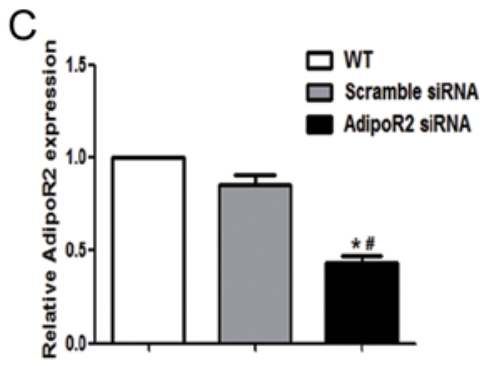

$\mathrm{F}$

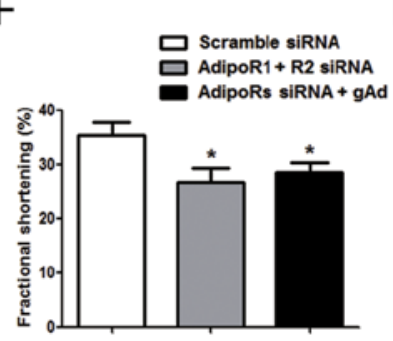

$\mathrm{E}$

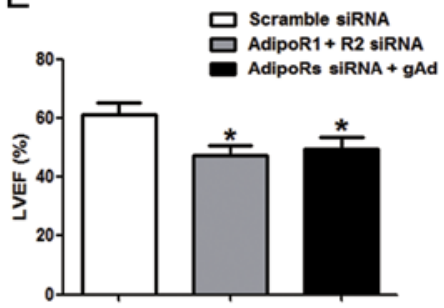

G

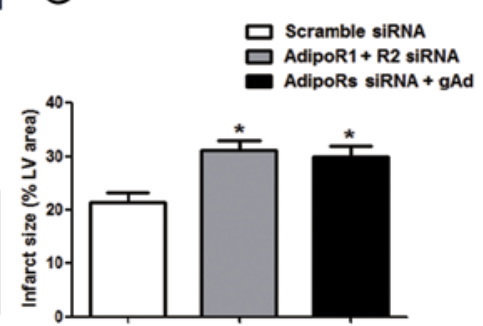

Figure 3

Protective effect of IPC was attenuated in AdipoR1 and AdipoR2 knockdown rats. (A) siRNA targening AdipoR1 and AdipoR2 were delivered by intramyocardial injection $10 \mathrm{~min}$ before IPC, the expression of myocardial AdipoR1 and R2 were tested by Western blot. Quantitative analysis of Western blot showed AdipoR1 expression (B) and AdipoR2 expression (C) ( ${ }^{*} P<0.05$ vs WT rats, $\# P<0.05$ vs scramble siRNA). (D) Representative echocardiography images in AdipoRs knock down rats showed the attenuated systolic function of left anterior wall (as arrow indicated), without improvement by gAd administration. (E) and (F) quantification of LVEF and FS from AdipoR knockdown or AdipoR knockdown with gAd administration rats by echocardiography 14 days after MI. (G) and (F) Myocardial infarct size in AdipoRs knockdown rats was showed by Masson's trichrome staining in AdipoR knockdown rats with or without gAd treatment and by quantitative analysis (\% left ventricular area). $n=6-8$ animals/group. ${ }^{*} P<0.05$ vs scramble siRNA, ${ }^{P} P<0.05$ vs IPC without siRNA. 
A
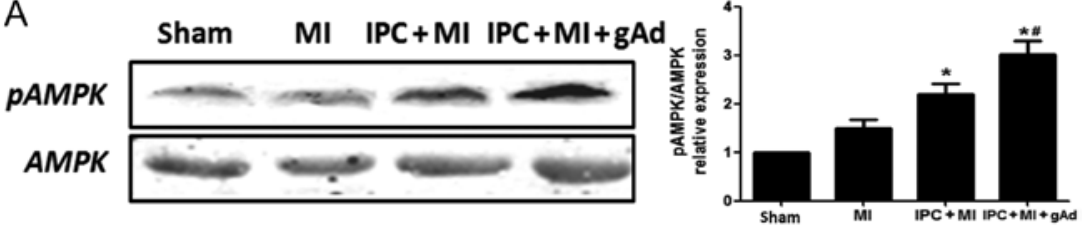

B

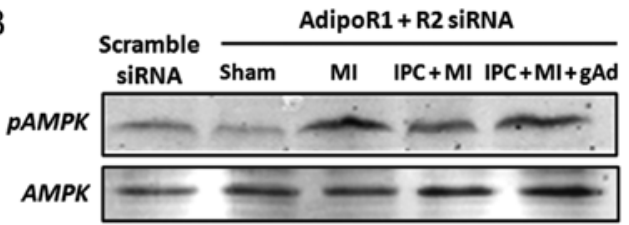

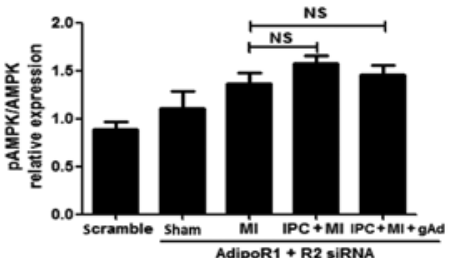

Figure 4

gAd treatment increased phosphorylation of AMPK (pAMPK) expression in the rat heart tissues 14 days after MI $24 \mathrm{~h}$ after sham operation or IPC, but without significant differences in AdipoRknockdown rats. (A) pAMPK expression in WT rats with IPC and $\mathrm{MI}$ and gAd treatment was tested by Western blot, and the quantification of Western blot was indicated by the PAMPK/AMPK ratio. (B) AdipoRs knockdown had no effect on the expression of PAMPK in heart tissues after IPC and $\mathrm{MI}$, also without changes after gAd administration, and the quantification of Western blot was indicated by the PAMPK/AMPK ratio. $n=6-8$ animals/group. ${ }^{*} P<0.05$ vs $\mathrm{MI}$ rats, $\# P<0.05$ vs IPC + MI rats.

\section{Discussion}

The beneficial effect of IPC on ischemic injury of cardiomyocytes has long been recognized, although the underlying mechanisms are not fully understood. In the present study, we obtained evidence to support the efficacy of IPC against myocardial ischemic injury and upregulated APN expression induced by IPC, thereby conferring the cardioprotective effects against MI injury. To our knowledge, we have demonstrated for the first time the causative relationship between IPC-induced APN upregulation and consequential cardioprotective effects. These results are consistent with our previous study concerning the induced expression of APN in ischemic myocardium and plasma by IPC and the relationship between APN and cardioprotective effects of IPC (Wang et al. 2010a).

Since 1986 when Murry and coworkers first described the phenomenon of IPC, researchers have paid close attention to its protection against myocardial ischemic injury induced by acute MI or myocardial ischemia/ reperfusion injury (Murry et al. 1986). Similar to the results of previous studies (Zheng et al. 2006, Eckle et al. 2008 ) that evaluated the effects of IPC on heart protection in animal models of MI, we found that the MI size was significantly smaller in IPC rats at 14 days after permanent ligation of the descending coronary artery compared with sham-operated rats. During the past 20 years, many studies have focused on IPC and its mechanisms. Several studies have suggested that the release of endogenous substances during preconditioning episodes plays an essential role in the protective effect of IPC. Adenosine, bradykinin, catecholamine and opioids are some of the substances released locally during IPC, which are of primary importance in mediating cardioprotection (Downey et al. 2005, 2007). Signaling pathways are activated by these factors combining with certain receptors through divergent pathways and activate the downstream signaling pathways of protein kinase C (Saurin et al. 2002, Simkhovich et al. 2013), p38 mitogenactivated protein kinase and AMPK (Lochner et al. 2009). The results of the present study support that APN may be one of the primary substances involved in protection by IPC via activation of the downstream signaling pathways of AMPK through its receptors.

Furthermore, we determined that IPC is responsible for the increased APN levels, thereby exerting cardioprotective effects against MI injury. There are many disputed clinical trials about the relationship between plasma APN levels and myocardial ischemic injury. De Roeck and coworkers found no significant relationship between circulating APN levels and ischemic reperfusion injury (De Roeck et al. 2016). However, most clinical trials show that low plasma APN levels are observed in patients with acute myocardial infarction (AMI), and those with the complication of hypoadiponectinemia are considered as a high-risk population for AMI events (Nakamura et al. 2004, Takashi et al. 2010). In addition, a prospective study has shown that high plasma APN levels are associated with a lower risk of MI (Pischon et al. 2004). In animal studies, APN possesses anti-inflammatory and antiischemic properties (Lau et al. 2011) that are significantly downregulated after myocardial ischemia/reperfusion injury (Wang et al. 2010b). APN-knockout mice manifest significantly enlarged infarct sizes and increased apoptosis during myocardial ischemia/reperfusion injury, which can be reversed by exogenous APN administration (Shibata et al. 2005). Although promising as a therapeutic agent for ischemic injury, APN only comprises about $0.01 \%$ of all plasma proteins. It would be clinically difficult to increase the concentration of APN by injecting purified protein. Thus far, few studies have reported on the effect of restoring the APN imbalance in animals http://jme.endocrinology-journals.org DOI: 10.1530/JME-16-0163
C 2017 Society for Endocrinology Printed in Great Britain
Published by Bioscientifica Ltd 
during myocardial ischemia/reperfusion injury without direct administration of APN. Therefore, it may be more effective to increase local expression of APN by specific mechanisms. Moreover, it has recently been recognized that APN expression is not solely restricted to adipocytes because cardiomyocytes also express APN (Pineiro et al. 2005, Ding et al. 2007). However, the expression pattern of cardiac-derived APN has not been defined after IPC. In our study, we found that IPC, which is at a mechanistic point upstream of the pathology responsible for APN elevation, upregulated cardiac-derived APN expression and its plasma concentration, which exhibited a timedependent change and reached its apogee at $12 \mathrm{~h}$ after IPC. Therefore, we present direct evidence that both systemically and locally derived APN levels vary after IPC, which may protect the heart from ischemic injury.

Previous studies have shown that insulin upregulates APN expression in visceral adipose tissue (Halleux et al. 2001), and the increased APN, once released into plasma, is capable of protecting ischemic hearts (Ouchi et al. 2006). In addition to abdominal fat, studies have suggested that epicardial fat may secrete a high amount of adipokines, mediating their physiological functions partly through a paracrine/autocrine manner. However, Fei and coworkers found that the expression of APN in epicardial fat increases with age, and histology cannot be performed because of the very low availability of epicardial fat tissue (Fei et al. 2010). Similar to their study, the 8 -week-old male rats had almost no epicardial fat in our study. Therefore, we did not investigate the physiological effects of APN from epicardial fat. The increased plasma APN after IPC in our study may also partly arise from adipose tissue, which is biologically active to protect the myocardium from ischemic injury. However, the specific role of cardiac-derived APN in mediating ischemic injury after IPC is unknown. To demonstrate that IPC upregulates cardiac-derived APN levels, which subsequently confer cardioprotective effects against MI injury, we performed in vivo siRNA-mediated knockdown of APN and AdipoRs via intramyocardial delivery to knockdown local APN, AdipoR1 and AdipoR2. AdipoR1 is abundantly expressed in skeletal muscle and cardiomyocytes, which has high affinity for globular APN. AdipoR2 is predominantly expressed in the liver and has high affinity for full-length APN. In addition to these receptors, T-cadherin is a putative cell-surfacebinding protein for full-length and oligomeric APN. It has been reported that both APN and its receptors are expressed in cardiomyocytes. Because APN-, AdipoR1- or AdipoR2-knockout rats were not commercially available, we used the siRNA-mediated gene silencing technique to knockdown APN and AdipoR expression in the rat heart. The results showed that the cardioprotective effects of IPC were abolished by siRNA-mediated repression of APN and AdipoR expression. Although increased APN levels by gAd treatment directly improved the protective effects in APN-knockdown rats, there were no significant changes in AdipoR-repressed rats, followed by inhibition of AMPK phosphorylation. Therefore, our study has revealed a central role of APN in myocardial IPC to increase myocardial resistance against subsequent ischemic tissue injury and suggests pharmacological strategies for APN activation in the treatment of AMI.

However, there is a major limitation using the siRNA-mediated gene-silencing technique in our study. The siRNA delivery only suppressed APN and AdipoR expression to $60 \%$ and $64 \%$, respectively, in knockdown animals, leading to incomplete blockade of APN signaling activation.

In summary, our study provides evidence for a novel mechanism of IPC that may improve the outcomes of ischemic patients. Thus, investigation of APN may provide unique insights into the cardioprotective effects of IPC and might lead to more therapeutic options for ischemic cardiovascular diseases. IPC results in changes in the levels of several circulating factors. Therefore, it will be important to perform further studies in large animals or clinical trials to elucidate the roles and interactions of these various factors in cardiovascular responses to IPC.

\section{Supplementary data}

This is linked to the online version of the paper at http://dx.doi.org/10.1530/ JME-16-0163.

\section{Declaration of interest}

The authors declare that there is no conflict of interest that could be perceived as prejudicing the impartiality of the research reported.

\section{Funding}

This work was supported by National Natural Science Foundation of China (No. 81170287).

\section{Acknowledgements}

The authors are grateful for the excellent technical assistance of Li Wang and Bo Liu from the Department of Physiology and Pathophysiology, Peking University, China. http://jme.endocrinology-journals.org DOI: 10.1530/JME-16-0163
(C) 2017 Society for Endocrinology Printed in Great Britain
Published by Bioscientifica Ltd 


\section{References}

Amin R, Mathews S, Alli A \& Leff T 2010 Endogenously produced adiponectin protects cardiomyocytes from hypertrophy by a PPARgamma-dependent autocrine mechanism. American Journal of Physiology: Heart and Circulatory Physiology 299 H690-H698. (doi:10.1152/ajpheart.01032)

Andersen A, Povlsen J, Bøtker H \& Nielsen-Kudsk J 2012 Ischemic preconditioning reduces right ventricular infarct size through opening of mitochondrial potassium channels. Cardiology $\mathbf{1 2 3}$ 177-180. (doi:10.1159/000342481)

Crisostomo P, Wairiuko G, Wang M, Tsai B, Morrell E \& Meldrum D 2006 Preconditioning versus postconditioning: mechanisms and therapeutic potentials. Journal of the American College of Surgeons 202 797-812. (doi:10.1016/j.jamcollsurg.2005.12.002)

De Roeck L, Vandamme S, Everaert B, Hoymans V, Haine S, Vandendriessche T, Bosmans J, Ronsyn M, Miljoen $\mathrm{H}$, Van BA, et al. 2016 Adiponectin and ischemia-reperfusion injury in ST segment elevation myocardial infarction. European Heart Journal: Acute Cardiovascular Care 5 71-76. (doi:10.1177/2048872615570770)

Ding G, Qin Q, He N, Francis-David S, Hou J, Liu J, Ricks E \& Yang Q 2007 Adiponectin and its receptors are expressed in adult ventricular cardiomyocytes and rpregulated by activation of peroxisome proliferator-activated receptor gamma. Journal of Molecular and Cellular Cardiology 43 73-84. (doi:10.1016/j.yjmcc.2007.04.014)

Downey J, Davis A \& Cohen M 2005 Unraveling the mysterious of classical preconditioning. Journal of Molecular and Cellular Cardiology 39 845-848. (doi:10.1016/j.yjmcc.2005.07.016)

Downey J, Davis A \& Cohen M 2007 Signaling pathways in ischemic preconditioning. Heart Failure Reviews 12 181-188. (doi:10.1007/ s10741-007-9025-2)

Eckle T, Kohler D, Lehmann R, Kasmi KE \& Eltzschig H 2008 Hypoxiainducible factor- 1 is central to cardioprotecion, a new paradigm for ischemic preconditioning. Circulation 118 166-175. (doi:10.1161/ CIRCULATIONAHA.107.758516)

Edelberg J, Lee S, Kaur M, Tang L, Feirt N, McCabe S, Bramwell O, Wong S \& Hong M 2002 Platelet-derived growth factor-AB limits the extent of myocardial infarction in a rat model: feasibility of restoring impaired angiogenic capacity in the aging heart. Circulation 105 608-613. (doi:10.1161/hc0502.103672)

Evrengul H, Seleci D, Tanriverdi H \& Kaftan A 2006 The antiarrhythmic effect and clinical consequences of ischemic preconditioning. Coronary Artery Disease 17 283-288. (doi:10.1097/00019501-200605000-00013)

Fei J, Cook C, Blough E \& Santanam N 2010 Age and sex mediated changes in epicardial fat adipokines. Atherosclerosis 212 488-494. (doi:10.1016/j.atherosclerosis.2010.06.044)

Fukuda S, Kaga S, Sasaki H, Zhan L, Zhu L, Otani H, Kalfin RK, Das D \& Maulik N 2004 Angiogenic signal triggered by ischemic stress induces myocardial repair in rat during chronic infarction. Journal of Molecular and Cellular Cardiology 36 547-559. (doi:10.1016/j. yjmcc.2004.02.002)

Goldstein BJ, Scalia RG \& Ma XL 2009 Protective vascular and myocardial effects of adiponectin. Nature Clinical Practice Cardiovascular Medicine 6 27-35. (doi:10.1038/ncpcardio1398)

Halleux C, Takahashi M, Delporte M, Detry R, Funahashi T, Matsuzawa Y \& Brichard S 2001 Secretion of adiponectin and regulation of apM1 gene expression in human visceral adipose tissue. Biochemical and Biophysical Research Communications 288 1102-1107. (doi:10.1006/bbrc.2001.5904)

Hotta K, Funahashi T, Arita Y, Takahashi M, Matsuda M, Okamoto Y, Iwahashi H, Kuriyama H, Ouchi N, Maeda K, et al. 2000 Plasma concentrations of a novel, adipose-specific protein, adiponectin, in type 2 diabetic patients. Arteriosclerosis, Thrombosis, and Vascular Biology 20 1595-1599. (doi:10.1161/01.ATV.20.6.1595)

Jia G, Yunfei B, Rui B, Hong L, Minghuan F \& Chuanshi X 2013 Globular adiponctin attenuate myocardial ischemia/reperfusion injury by upregulating endoplasmic reticulum $\mathrm{Ca}^{2+}-$ ATPase activity and inhibiting endoplasmic reticulum stress. Journal of Cardiovascular Pharmacology and Therapeutics 62 143-153. (doi:10.1097/ FJC.0b013e31829521af)

Lau W, Tao L, Wang Y, Li R \& Ma XL 2011 Systemic adiponectin malfunction as a risk factor for cardiovascular disease. Antioxidants and Redox Signaling 15 1863-1873. (doi:10.1089/ars.2010.3743)

Ling T, Erhe G, Xiangying J, Yuexing Y, Shuzhuang L, Theodore AC, Bernard LL, Walter K, Lawrence C, Barry JG, et al. 2007 Adiponectin cardioprotection after myocardial ischemia/ reperfusion involves the reduction of oxidative/nitrative stress. Circulation 115 1408-1416. (doi:10.1161/CIRCULATIONAHA. 106.666941)

Lochner A, Marais E, Genade S, Huisamen B, Du Toit E \& Moonman J 2009 Protection of the ischaemic heart: investigation into the phenomenon of ischaemic preconditioning. Cardiovascular Journal of Africa 20 43-51.

Murry CE, Jennings RB \& Reimer KA 1986 Preconditioning with ischemia: a delay of lethal cell injury in ischemic myocardium. Circulation 74 1124-1136.

Nakamura Y, Shimada K, Fukuda D, Shimada Y, Ehara S \& Hirose M 2004 Implications of plasma concentrations of adiponectin in patients with coronary artery disease. Heart 90 528-533. (doi:10.1136/hrt.2003.011114)

Ouchi N, Kobayashi H, Kihara S, Kumada M, Sato K, Inoue T, Funahashi T \& Walsh K 2004 Adiponectin stimulates angiogenesis by promoting cross-talk between AMP-activated protein kinase and Akt signaling in endothelial cells. Journal of Biological Chemistry 279 1304-1309. (doi:10.1074/jbc.M310389200)

Ouchi N, Shibata R \& Walsh K 2006 Cardioprotecion by adiponectin. Trends in Cardiovascular Medicine 16 141-146. (doi:10.1016/j. tcm.2006.03.001)

Pineiro R, Iglesias M, Gallego R, Raghay K, Eiras S, Rubio J, Diéguez C, Gualillo O, González-Juanatey J \& Lago F 2005 Adiponectin is synthesized and secreted by human and murine cardiomyocytes. FEBS Letters 579 5163-5169. (doi:10.1016/j.febslet.2005.07.098)

Pischon T, Girman C, Hotamisligil G, Rifai N, Hu F \& Rimm E 2004 Plasma adiponectin levels and risk of myocardial infarction in men. JAMA 291 1730-1737. (doi:10.1001/jama.291.14.1730)

Raghu A, Bee KT, Jing C \& Harpal SR 2012 Protective actions of globular and full-length adiponectin on human endothelial cells: novel insights into adiponectin-induced angiogenesis. Journal of Vascular Research 49 534-543. (doi:10.1159/000338279)

Saurin A, Pennington D, Raat N, Latchman D, Owen M \& Merber M 2002 Targeted disruption of the protein kinase $\mathrm{C}$ epsilon gene abolishes the infarct size reduction that follows ischaemic preconditioning of isolated buffer-perfused mouse hearts. Cardiovascular Research 55 672-680. (doi:10.1016/S00086363(02)00325-5)

Shibata R, Ouchi N, Kihara S, Sato K, Funahashi T \& Walsh K 2004 Adiponectin stimulates angiogenesis in response to tissue ischemia through stimulation of AMP-activated protein kinase signaling. Journal of Biological Chemistry 279 28670-28674. (doi:10.1074/jbc. M402558200)

Shibata R, Sato K, Pimentel DR, Takemura Y, Kihara S, Ohashi K, Funahashi T, Ouchi N \& Walsh K 2005 Adiponectin protects against myocardial ischemia-reperfusion injury through AMPK- and COX-2dependent mechanisms. Nature Medicine 11 1096-1103. (doi:10.1038/nm1295)

Simkhovich B, Przyklenk K \& Kloner R 2013 Role of protein kinase C in ischemic 'conditioning': from first evidence to current perspectives. Journal of Cardiovascular Pharmacology and Therapeutics 18 525-532. (doi:10.1177/1074248413494814)

Song Y, Yu Q, Zhang J, Huang W, Liu Y, Pei H, Liu J, Sun L, Yang L, Li C, et al. 2014 Increased myocardial ischemia-reperfusion injury in renal failure involves cardiac adiponectin signal deficiency. American

Published by Bioscientifica Ltd. 
Journal of Physiology: Endocrinology and Metabolism 306 E1055-E1064. (doi:10.1152/ajpendo.00428.2013)

Sugano M, Tsuchida K, Hata T \& Makino N 2005 RNA interference targeting SHP-1 attenuates myocardial infarction in rats. FASEB Journal 19 2054-2056. (doi:10.1096/fj.05-4020fje)

Takashi H, Mao T, Takuo L \& Kohji S 2010 Relationship between two AMI risk factors: low serum lipoprotein lipase mass and hypoadiponectinemia. Journal of Atherosclerosis and Thrombosis $\mathbf{1 7}$ 001-009. (doi:10.1161/ATVBAHA.108.181164)

Thirunavukkarasu M, Han Z, Zhan L, Penumathsa S, Menon V \& Maulik N 2008 Adeno-sh- $\beta$-Catenin abolishes ischemic preconditioning-mediated cardioprotection by downregulatioin of its target genes VEGF, Bcl-2, and Survivein in ischemic rat myocardium. Antioxidants and Redox Signaling 10 1475-1485. (doi:10.1089/ ars.2008.2042)
Wang H, Cheng J, Wu W, Wen J, Liao V, Kong W, Ke Y \& Zheng J 2010a Ischemia preconditioning attenuated myocardial ischemia via upregulating the expression of adiponectin in the rat. Zhongua Xin Xue Guan Bing Za Zhi 38 929-933. (doi:10.3760/ cma.j.issn.0253-3758.2010.10.014)

Wang Y, Lau W, Gao E, Tao L, Yuan Y, Li R, Wang X, Koch W \& Ma X $2010 b$ Cardiomyocyte-derived adiponectin is biologically active in protecting against myocardial ischemia-reperfusion injury. American Journal of Physiology: Endocrinology and Metabolism 298 E663-E670. (doi:10.1152/ajpendo.00663.2009)

Zheng J, Chin A, Duignan I, Won K, Hong M \& Edelberg J 2006 Growth factor-mediated reversal of senescent dysfunction of ischemiainduced cardioprotection. American Journal of Physiology: Heart and Circulatory Physiology 290 H525-H530. (doi:10.1152/ ajpheart.00470.2005)

Received in final form 25 January 2017

Accepted 27 January 2017

Accepted Preprint published online 20 February 2017 http://jme.endocrinology-journals.org DOI: 10.1530/JME-16-0163
(C) 2017 Society for Endocrinology Printed in Great Britain
Published by Bioscientifica Ltd. 